\title{
news
}

IN this section
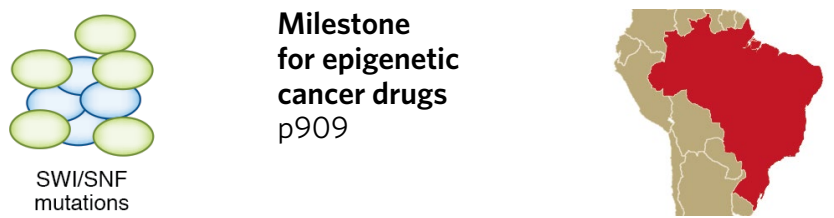

Biotech news from around the globe p912

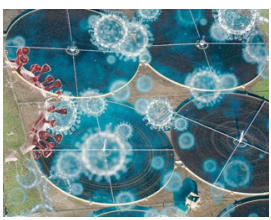

Using wastewater to monitor SARS-CoV-2 p917

\section{Focus shifts to antibody cocktails for COVID-19 cytokine storm}

Emerging clinical trial data suggest that individual immunomodulatory drugs can dampen the hyperactive immune system in severe COVID-19, but polytherapy is the way forward.

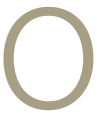

$\mathrm{n}$ June 5 the Ministry of Health of the Russian Federation registered Biocad's Ilsira (levilimab) as a treatment for severe COVID-19. Ilsira is one of several monoclonal antibodies (mAbs) targeting interleukin-6 receptor (IL-6R) and a host of other inflammatory cytokines currently in clinical testing against coronavirus. As Nature Biotechnology went to press, peer-reviewed data assessing the efficacy of these treatments were limited: a small clinical study of Roche's Actemra (tocilizumab, an mAb targeting IL-6R) and an open-label trial of Bermuda-based Kiniksa Pharmaceuticals' mavrilimumab (a fully human IgG4 mAb targeting granulocytemacrophage colony stimulating factor, GM-CSF). As clinicians await published data from a raft of other IL-6 and GM-CSF inhibitors in human testing, the need to combine agents targeting different cytokines is emerging as the likely path forward for supportive care for COVID-19 patients with acute respiratory distress syndrome.

Ilsira's Russian approval follows the announcement in March that China's National Health Commission would register Actemra for use in patients with coronavirus infection who have serious lung lesions and high IL-6 levels. As of mid-July, data from the Ilsira trial used to support the mAb's approval remained unpublished, whereas for Actemra, one peer-reviewed paper details results in a trial setting. Although the mAb improved survival and outcomes in patients undergoing ventilation, its effectiveness remains much less clear in patients with less severe disease; so far, it remains unproven whether Actemra reduces patient admissions to intensive-care units, the need for ventilation, or hospitalization times. Some of these questions may be addressed in Roche's phase 3 COVACTA trial, which is enrolling hospitalized patients with varying COVID-19 severities; with a readout at the end of August,

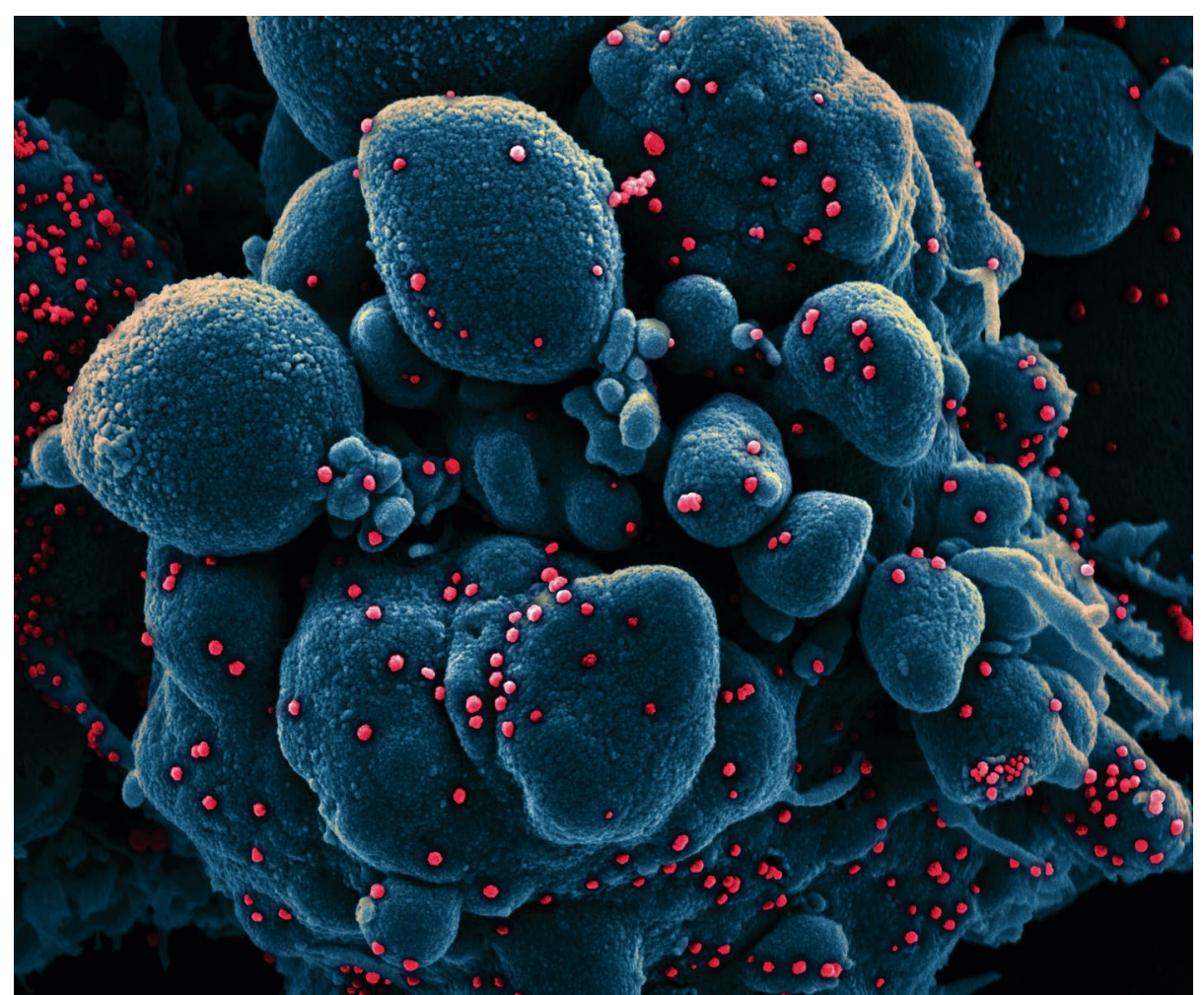

The new coronavirus SARS-CoV-2 (red) infects a patient's cells (blue). In certain cases, this infection can induce an excessive immune response with pro-inflammatory cytokines known as a 'cytokine storm'. Credit: NIAID/NIH / Science Source

this trial is expected to provide further clarity about the effectiveness of IL- 6 modulation in clinical practice.

Actemra was one of the first drugs tested in severe COVID-19. It has a US Food and Drug Administration approval to treat cytokine release syndrome in patients undergoing CAR-T cell therapy for cancer, which mirrors clinical features of severe COVID-19. In many patients who become critically ill, the SARS-COV-2 virus triggers a hyperinflammatory response characterized by excessive cytokine release, often referred to as a 'cytokine storm'. Molecules such as interleukin (IL)-6, IL-1, IL-2, IL-7, IL-10, interferon (IFN) $-\gamma$ inducible protein, monocyte chemoattractant protein (MCP)1 , macrophage inflammatory protein $1 \mathrm{~A}$ and tumor necrosis factor (TNF)- $\alpha$ cause widespread damage, with the cytokine storm proportional to the severity of COVID-19. When produced in huge quantities, these 
Table 1 | Peer-reviewed evidence for clinical efficacy in COVID-19 of anti-IL-6, anti-IL-1, anti-GM-CSF and anti-TNF mAbs alone and in combination, as of 9 July

\section{Drug/company}

\section{Anti-IL-6 drugs}

Ilsira (levilimab)/Biocad

Kevzara (sarilumab)/Regeneron, Sanofi

Sylvant (siltuximab)/EUSA Pharma,

BeiGene

Actemra (tocilizumab)/Roche 814-818(2020); Sciascia et al. Clin. Exp. Rheumatol. 38, 529-532 (2020); Kewan et al. EClinicalMedicine (18 June 2020); (24 June 2020)

Olokizumab (R-Pharm)

Clazakizumab/Bristol-Myers Squibb, Alder IL-6 Biopharmaceuticals

\section{Anti-IL-1 drugs}

Kineret (anakinra)/Novartis, Swedish Orphan Biovitrum

Ilaris (canakinumab)/Novartis

RPH-104 (fusion comprising IL-1R1 and IL-1RAcP linked to IgG heavy chains)/R-Pharm

\section{Anti-GM-CSF drugs}

Mavrilimumab/Kiniksa

Lenzilumab (an engineered

non-fucosylated recombinant human IgG1 mAb)/Humanigen

TJ003234 (a humanized IgG1 mAb)/I-Mab

Gimsilumab/Roivant

Otilimab/MorphoSys, GlaxoSmithKline

Namilumab/Izana

\section{Anti-TNF- $\alpha$ drugs}

XPro1595/INmune Bio

Gamifant (emapalumab)/Swedish Orphan Biovitrum

Remicade (infliximab)/Janssen
IL-6R

Luo et al. J. Med. Virol. 37, Guaraldi et al. Lancet Rheumatol.

$\begin{array}{ll}\text { IL-6R } & \text { N.A. } \\ \text { IL-6R } & \text { Bennuci et al. J. Med. Virol. }\end{array}$ (30 May 2020); Della-Torre et al. Ann. Rheum. Dis. (3 July 2020)

N.A.

N.A.

N.A.

IL-1 Cavalli et al. Lancet Rheumatol. 2, e325-e331 (7 May 2020)

IL-1 $\beta \quad$ N.A.

IL-1 $\beta \quad$ N.A.

GM-CSF De Luca et al. Lancet Rheumatol. (16 June 2020)

GM-CSF N.A.

GM-CSF N.A.

GM-CSF N.A.

GM-CSF N.A.

GM-CSF N.A.

TNF- $\alpha \quad$ N.A.

TNF- $\alpha \quad$ N.A.

TNF- $\alpha \quad$ N.A.

\section{Trial type, data readout/polytherapy or monotherapy}

Phase 3 (NCT04397562)/monotherapy

Phase 3, suspended (NCT04327388)/monotherapy

Phase 3 (NCT04330638), readout end of September/ monotherapy

Phase 3 (NCT04330638), readout end of September/ polytherapy with Kineret

Phase 3 (NCT04320615), readout end of August/ monotherapy

Phase 3 (NCT04330638), readout end of September/ polytherapy with Kineret

Phase 2/3 (NCT04380519), readout mid-October/ monotherapy

Phase 2 (NCT04348500), readout end of December/ monotherapy

Phase 2 (NCT04363502), readout end of August/ monotherapy

Phase 2 (NCT04343989), readout beginning July/ monotherapy

Phase 3 (NCT04364009), readout mid-September/ monotherapy

Phase 3 (NCT04362813), readout end of July/ monotherapy

Phase 2/3 (NCT04380519), readout mid-October/ monotherapy

Phase 2 (NCT04399980), readout end of May 2021/ monotherapy

Phase 3 (NCT04351152), readout September/ monotherapy

Phase 1/2 (NCT04341116), readout September/ monotherapy

Phase 2 (NCTO4351243), readout October/ monotherapy

Phase 2 (NCTO4376684), readout mid-December/ monotherapy

Part of CATALYST trial/monotherapy

Phase 2/3 (NCT04370236), readout February 2021 (not yet recruiting)/monotherapy

Phase 2/3 (NCT04324021), readout July/monotherapy

Phase 2 (NCT04425538), readout September/ monotherapy

Part of CATALYST trial/monotherapy 


\section{Box 1 | Blood profiling and COVID-19 severity prediction}

Blood profiling may make it possible to differentiate between degrees of COVID-19 severity. Two recent studies in Cell and Cell Systems identified proteomic markers that achieve this, including markers linked to complement and coagulation, macrophage function, platelet degranulation and modulation of inflammation. But identifying a COVID-19-specific biomarker could prove tricky, as COVID-19 shares response profiles with several other viral infections.

Immunological markers that measure T-cell and/or B-cell dysregulation in COVID-19 patients, for instance, might be one route to that elusive specificity. A paper in Immunity from Mascha Binder's group at Martin Luther University of Halle-Wittenberg in Germany looked at B-cell and T-cell receptor sequences from cells isolated from the blood of 383 COVID-19 patients with active infection or after recovery. TCR repertoires were found to skew not only to those associated with IFN type I and III responses and early $\mathrm{CD}^{+}{ }^{+}$and $\mathrm{CD} 8^{+} \mathrm{T}$-cell activation, but also to $\mathrm{T}$ follicular helper cells, $\mathrm{T}$ helper 17 $\left(\mathrm{T}_{\mathrm{H}} 17\right)$-like and non-conventional (but not classic anti-viral) $\mathrm{T}_{\mathrm{H}} 1$ cell polarizations. Overall, 31 TCR clusters emerged with potential as predictors of protective immunity. Although provisional, the analysis also indicates that patients with $B$ lineage repertoires exhibiting a high degree of somatic hypermutation may also be at higher risk for severe disease.

Elsewhere, two preprints, posted by teams led by groups at the University of Pennsylvania and London's Crick Institute, also hint at possible immune signatures' linked to distinct COVID-19 disease progression and outcomes, despite patient heterogeneity. The studies used flow cytometry to assess T- and B-cell populations in blood samples from patients, as well as recovered and healthy individuals.

The Crick study found reduction in a specific T-cell population $\left(\mathrm{CD}^{+} \mathrm{T}_{\mathrm{H}} 17.1\right.$ cells, which produce IFN- $\gamma$ ) in patients with severe COVID-19. The paper's lead author, Adrian Hayday, notes that T-cell activity in COVID-19 might be restored by promoting particular cytokines - for instance, IL-7. A phase 2 trial administering IL-7 to patients with COVID-19 and lymphopenia is in progress, sponsored by RevImmune. There's still a way to go in deciphering the underlying biology: "Nobody yet understands how these T cells are getting knocked out," says Hayday.

Elsewhere, Adaptive Biotechnologies has a large study monitoring patients' $\mathrm{T}$ cell repertoire responses to cancer treatments and is now using its platform to probe the T-cell biology of COVID-19. In partnership with Microsoft, the company is profiling the T-cell receptor (TCR) repertoire involved in the response to SARS-CoV-2 infection; each sequence acts as a unique tag or barcode that can be used to follow a particular T-cell clone. According to Adaptive's CSO Harlan Robins, the platform can "see the dynamics of a particular clone over time", such as when it proliferates and whether it persists or goes away, he adds. The first data from this project were released by the company on 11 June.

Similar efforts are under way across the community to pool data, including the notable iReceptor COVID-19 initiative. All this will be helpful, according to Merad. "We should not hesitate to investigate as deep as possible," she says. pro-inflammatory cytokines, particularly IL-6, generally correlate with respiratory failure and mortality.

Elsewhere, several other mAbs targeting IL-6 are progressing through testing, including R-Pharm's olokizumab, a humanized anti-IL- $6 \mathrm{mAb}$, and Sylvant (siltuximab), an anti-IL-6 chimeric IgG1 $\mathrm{mAb}$ from EUSA Pharma and BeiGene. Yet Sanofi and Regeneron shut down a US-based phase 3 trial with Kevzara (sarilumab, a human IgG1 mAb targeting IL-6R approved in rheumatoid arthritis) after the drug failed to improve survival in hospitalized patients or reduce their need for ventilation.

Although IL-6 inhibitors have thus far yielded mixed results, Miriam Merad, director of the Precision Immunology Institute at Mount Sinai School of Medicine, New York, says, "I will not give up on IL-6 - because it is so prominent," as she refers to findings that IL-6 is robustly and consistently increased across studies.

An idea gathering traction by Merad and others is to combine IL- 6 blockers with other cytokine-targeting antibodies (Table 1). "Biologic cocktails will be important" to target the multiple pathogenic factors involved in the hyperinflammation, says Cezmi Akdis at the Swiss Institute of Allergy and Asthma Research, University of
Zurich. His rationale is that tackling severe COVID-19 should be identical to tackling other disorders, such as sepsis, caused by a dysregulated immune system, "because the underlying mechanism is the same," he says.

Such cocktails, Akdis suggests, could combine the following: IL-6 inhibitors with the IL-1 receptor blocker Kineret (anakinra), a fusion protein from Novartis and Swedish Orphan Biovitrum comprising the human IL-1 type 1 receptor (extracellular domain and accessory protein) and the Fc portion of human IgG1; one of several TNF- $\alpha$-targeted mAbs on the market, such as Remicade (infliximab) or Enbrel (etanercept); and Gamifant (emapalumab), which binds IFN- $\gamma$.

An alternative strategy could be to boost a patient's supply of IFN. This is not widely embraced to treat infections because IFNs can cause tissue damage once viral infection is established. But SARS-CoV-2 might be unique among viruses in its ability to knock down the host's IFN levels to evade its host's innate immune system. A study published in Cell shows that SARS-CoV-2 in cell lines drives an inflammatory response characterized by low type I and type III IFN production alongside increased chemokine and IL-6 release. Other common respiratory viruses, including the coronaviruses Middle East respiratory syndrome coronavirus (MERS-CoV) and
SARS-CoV, human parainfluenza virus 3, respiratory syncytial virus and influenza $A$ virus, didn't follow this pattern.

Merad notes that clinical data to back this finding are mounting and raise the possibility of giving patients a type III IFN - receptor expression for which is restricted to only certain cells, thus lowering the risk of unwanted pathogenic effects while blocking IL- 6 and TNF- $\alpha$. "I would really try to privilege such combination blockades," she says, "because we are trying to target a complex inflammation process."

Although such combinations remain some way off, anticytokine monotherapies, as well as polytherapies with cocktails of IL-6 and IL-1 blockers, are already in testing. University Hospital Ghent in Belgium is trialing two combinations, Actemra with Kineret and Sylvant with Kineret, as part of a phase 3 trial.

An alternative to multiple cytokine blockade is to target a master controller of the immune system: granulocytemacrophage colony-stimulating factor (GM-CSF). GM-CSF is secreted during inflammation and stimulates IL-1, IL-6 and TNF- $\alpha$ production. Notably, in COVID-19 patients, levels of GM-CSF are high. As of July, peer-reviewed results of a phase $2 / 3$ trial of Kiniksa's mavrilimumab place 


\section{COVID vaccine results pour in}

On 20 July, results from clinical trials of two early-stage COVID-19 vaccines, one developed by University of Oxford and AstraZeneca and another by the Chinese company CanSino Biologics, were reported in the Lancet. Oxford's simian adenovirus-vectored vaccine (ChAdOx1 nCoV-19) and CanSino's adenovirus-5-vectored vaccine both elicited neutralizing antibodies and T-cell immune responses to the SARS-CoV-2 virus. (CanSino, based in Tianjin, was the first vaccine developer to publish data from a smaller phase 1 trial, in May.) These publications join Moderna's mRNA vaccine report from mid-July in the New England Journal of Medicine. Because of different assays used, comparisons among the vaccines are difficult. In broad terms, the two adenovirus-vectored programs gave similar results - upwards of 80-90\% of participants marshalled neutralizing antibody and spike-protein-specific T cell responses within 28 days - whereas the Moderna vaccine study reported only neutralizing antibody responses after vaccination. In the single-blind, randomized, placebo-controlled Oxford trial, immune responses lasted the full 56 days of the trial. The researchers inoculated a small cohort of ten people with a second dose of the COVID-19 vaccine after 28 days, which upped the antibody response but not the T-cell one, in keeping with previous observations. Participants in both trials had only mild flu-like symptoms, typically seen with viral-vector-mediated vaccines.

Although the results are promising, both trials involved relative young and ethnically homogenous people; neither of these represent demographics most affected by COVID-19. More diverse populations are included in Cambridge-based AstraZeneca's phase 3 trials of ChAdOx1 nCoV-19 taking place in South Africa and Brazil. Also on 20 July, Pfizer and German company BioNtech of Mainz published early results in an online preprint server of their mRNA vaccine candidate BNT162b1, showing dose-dependent neutralizing antibody responses and $\mathrm{CD} 4^{+}$and $\mathrm{CD} 8^{+} \mathrm{T}$ cell responses against the receptor-binding domain of SARS-CoV-2. Ultimately, though, although all of these potential vaccines have shown to engage the immune system, no vaccine developer has yet provided definitive data demonstrating protection from SARS-CoV-2 infection.

Published online: 5 August 2020

https://doi.org/10.1038/s41587-020-0647-4 the drug as a frontrunner among the anti-GM-CSF mAbs under development. Mavrilimumab eliminated deaths in the treated group, with $100 \%$ attaining clinical improvement by day 28 , as compared with only $65 \%$ in the comparison group. According to a presentation planned for the European League Against Rheumatism (EULAR) 2020 Digital Congress in August, Kiniksa is moving forward on the basis of this data with a multicenter, double-blind, randomized, placebo-controlled study.

Although GM-CSF blockade is an area of high trial activity, few results have as yet been published in the peer-reviewed literature. Other anti-GM-CSF antibodies in clinical trials for COVID-19 include Humanigen's lenzilumab (an engineered, non-fucosylated recombinant human IgG1 $\mathrm{mAb}$ ), which is in phase 3 trials, and Shanghai-based I-Mab's TJ003234 (a humanized IgG1 mAb), which is in phase 2 testing. Three other fully human IgG1 mAbs against GM-CSF are undergoing efficacy testing for COVID-19: Basel, Switzerland-based Roivant's gimsilumab; otilimab, from Planegg, Germany-based MorphoSys and Brentford, UK-based GlaxoSmithKline; and Oxford-based Izana's namilumab.

All things considered, clinical management of COVID-19 will likely require several different therapeutic strategies. "The idea that one biological therapy may turn out to be superior to others is probably not accurate" says Simon Jones of the Systems Immunity Research Institute, School of Medicine at Cardiff University in the UK. Different patients with the same underlying condition, such as rheumatoid arthritis, often show differing responses to specific biological therapies. "This is also probably reflected in patients with COVID-19," he says.

As an adjunct to these biologic approaches, small molecules have the advantage of being able to inhibit several cytokines at once. Cambridge, UK-based AstraZeneca's Calquence (acalabrutinib), approved for treating chronic lymphocytic leukemia, targets Bruton's tyrosine kinase (BTK), which is upstream of several inflammatory cytokines, such as IL-1, IL- 6 , and TNF- $\alpha$. An off-label trial published in Science Immunology showed that Calquence reduced patients' requirement for oxygen and normalized markers of inflammation. Levels of BTK were elevated in peripheral blood monocytes isolated from patients with COVID-19 compared with cells from healthy volunteers. A possible advantage to targeting BTK is that it is "specific for the type of cell - the macrophage - that is a leading cause of the cytokine surge," says Louis Staudt at the Center for Cancer Research at the US National Cancer Institute, an author on the publication. As such,
Calquence might have fewer side effects than other multi-cytokine blockers, such as JAK-STAT inhibitors, currently in clinical trials that modulate cytokine activity downstream of their receptors. On the basis of this result, AstraZeneca plans to test Calquence in larger trials.

If the preferred strategy is to tackle many cytokines at once, why not use steroids? Indeed, the steroid dexamethasone reduced deaths by one-third in patients on a ventilator and by one-fifth in patients receiving oxygen, as reported in a preprint in June from the large RECOVERY trial. However, dexamethasone only made a difference to patients seriously ill with COVID-19; in individuals not sick enough to be on respiratory support, dexamethasone did not improve survival. The aim of a therapy should be to "prevent people getting to ventilator stage, before damage such as organ disfunction, clotting disorders and secondary infections manifest," says Wyndham Wilson, senior investigator at the US National Cancer Institute. He adds that dexamethasone might be affecting inflammation within the lung itself and still do little to dampen the cytokine storm. When to intervene with a sledgehammer approach, such as dexamethasone, is just as critical. Starting a treatment that stifles the immune system too early in the disease course runs the risk of promoting viral replication because the very cytokines that are part of the surge also are vital in the host's defense against the virus.

To track when a patient's inflammatory response becomes life-threatening can be done with routine clinical tests, such as measurements of ferritin or C-reactive protein. And measuring IL-6 is relatively straightforward. But these types of analyses give only a blurry picture. "They are an index of inflammation that don't really tell us much about the direction of the pathology", says Jones. Markers, of say, endothelial damage, abnormal coagulation or lymphopenia may be better indicators of severe disease, he adds. In this respect, blood profiling and immune cell and biomarker studies may provide a clearer picture (Box 1).

Overall, the path to therapeutic success in COVID-19 will lie somewhere between stopping the hyperinflammation before the patient becomes very sick and allowing the body to eliminate persisting virus. "We're trying to do two things at the same time" says Merad. "This is a conundrum we are all facing."

\section{Charlotte Harrison \\ Canterbury, UK}

Published online: 5 August 2020

https://doi.org/10.1038/s41587-020-0634-9 